\title{
High expression of Tob1 indicates poor survival outcome and promotes tumour progression via a Wnt positive feedback loop in colon cancer
}

\author{
Dandan $\mathrm{Li}^{1,2+}$, Li Xiao ${ }^{2,3 \dagger}$, Yuetan Ge $\mathrm{G}^{2,4}$, Yu Fu², Wenqing Zhang ${ }^{2}$, Hanwei Cao ${ }^{2}$, Binbin Chen², Haibin Wang ${ }^{5,6^{*}}$,
} Yan-yan Zhan ${ }^{2^{*}}$ and Tianhui $\mathrm{Hu}^{1,2^{*}}$ (D)

\begin{abstract}
Tob1, a Tob/BTG anti-proliferative protein family member, functions as a tumour suppressor in many cancers. Here, we reveal a unique oncogenic role of Tob1 in colon cancer. Tob1 expression was upregulated during colon cancer progression, was significantly correlated with tumour size and tumour differentiation, and was a prognostic indicator of colon cancer. Unlike in other cancers, where nuclear Tob1 performs anticancer activity, Tob1 is predominantly localized in the cytosol of colon cancer cells, where this protein binds and stabilizes $\beta$-catenin to activate Wnt/ $\beta$-catenin signalling, which in turn enhances Tob1 expression, thus forming a positive feedback loop to promote cell proliferation. Moreover, Tob1 deficiency led to reduced tumourigenesis in AOM/DSS-treated and $\mathrm{ApC}^{\mathrm{Min} /+}$ mice. Our findings provide important insights into a previously unrecognized oncogenic role of Tob1 in colon cancer and suggest that Tob1 is an adverse prognostic factor and therapeutic target for colon cancer.
\end{abstract}

Keywords: Tob1, Tumour-promoting, Colon cancer, Prognostic marker, Wnt/ß-catenin signaling

\section{Main text}

Tob1, a Tob/BTG anti-proliferative protein family member, acts as a tumour suppressor in many cancers [1-3], though it might have oncogenic role in estrogen-independent ER-positive breast cancer cells [4]. In colon cancer cells, Tob1 was simply reported to be upregulated by EZH2 depletion [5]. In this study, we unexpectedly found that Tob1 acts as an oncogenic protein in colon cancer via a Wnt positive feedback loop.

\footnotetext{
* Correspondence: haibin.wang@vip.163.com; yyzhan@xmu.edu.cn; thu@xmu.edu.cn

${ }^{\dagger}$ Dandan Li and Li Xiao contributed equally to this work.

${ }^{5}$ Reproductive Medical Center, The First Affiliated Hospital of Xiamen University, Xiamen 361003, Fujian Province, People's Republic of China

${ }^{2}$ Cancer Research Center, Xiamen University Medical College, Xiamen 361102, Fujian Province, People's Republic of China

'Xiamen Cancer Hospital, The First Affiliated Hospital of Xiamen University,

Xiamen 361003, Fujian Province, People's Republic of China

Full list of author information is available at the end of the article
}

\section{Tob1 is upregulated in colon cancer and confers poor outcomes}

The Oncomine database [6] was analysed to compare Tob1 DNA and mRNA levels between colon cancer and normal tissues. The Kurashina and TCGA colorectal-2 datasets showed increased Tob1 DNA copies in colon cancer (Additional file 1: Table S1). Kaiser's datasets showed that Tob1 mRNA levels were significantly elevated in colon adenocarcinoma (Fig. 1A). Tob1 mRNA (Additional file 1: Figure S1A-B) and protein (Fig. 1B-C) levels were upregulated in colon cancer tissues compared to paired noncancerous tissues; however, Tob1 phosphorylation [7] did not differ between tumour and non-tumour tissues when normalized to Tob1 expression (Additional file 1: Figure S2). Tissue Microarray analysis of 84 colon cancer samples confirmed that Tob1 expression was significantly higher in colon cancer than in normal tissues $(P<0$. 001, Fig. 1D-E). Tob1 was mainly localized in the cytoplasm of both normal colonic epithelial and colon cancer cells (Fig. 1D), unlike the localization observed in other kinds of cancers or 

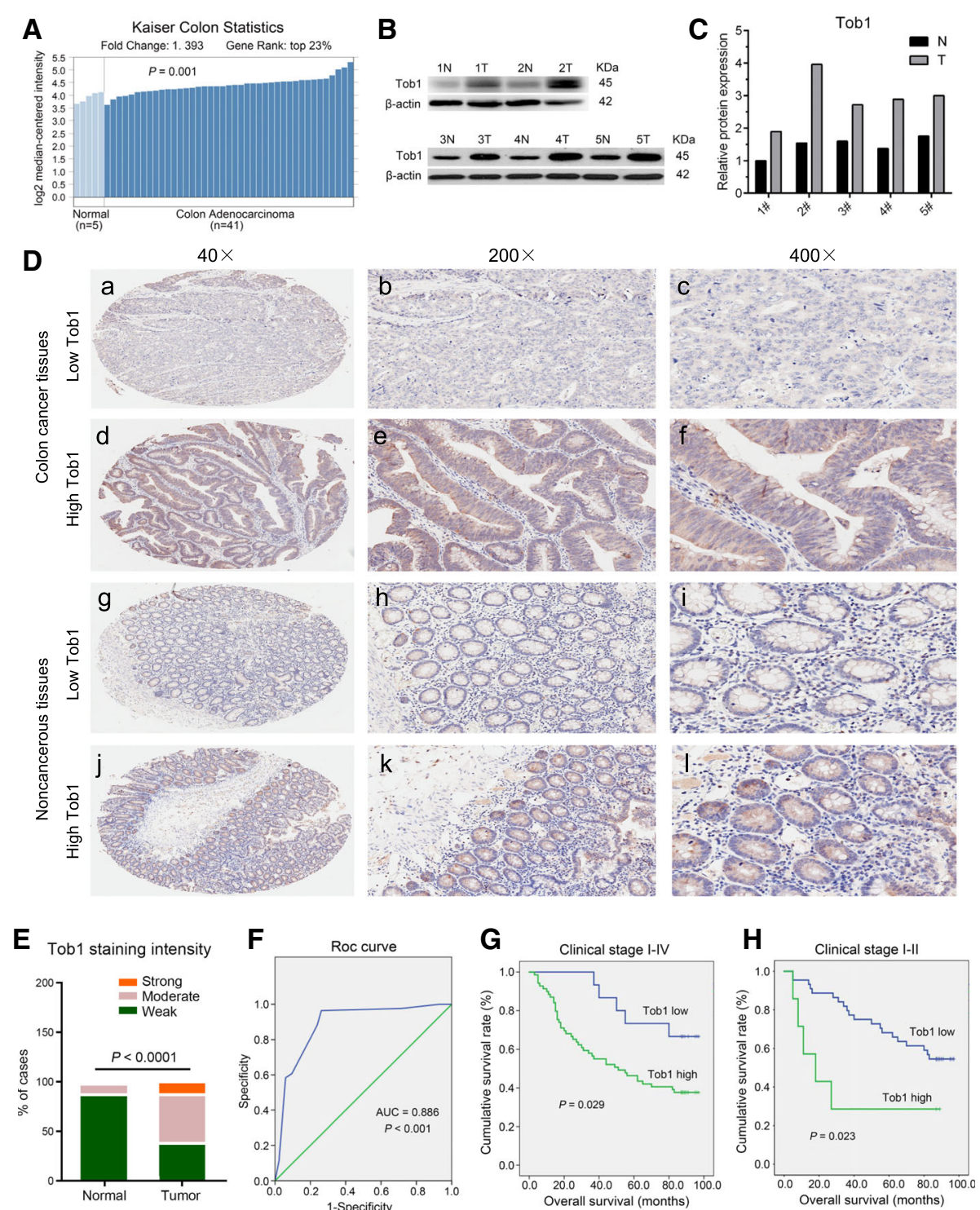

Fig. 1 Tob1 is upregulated in colon cancer. a Tob1 expression in Kaiser's colon cancer dataset from Oncomine. b-c Tob1 levels in colon cancer by WB. "N"-normal, "T"-tumour. Tob1 levels were normalized to $\beta$-actin levels. "T" vs " $N$ ", $n=5, P=0.008$, Mann-Whitney $U$ test. $\mathbf{d}$-e Tob1 expression was analysed by IHC based on a TMA containing 84 colon cancer specimens. $\mathbf{f}$ ROC curve for Tob1. g-h Kaplan-Meier OS curves for patients with different Tob1 expression levels

normal tissues [1-3]. ROC curve analysis provided the AUC (0.886) and IHC cut-off score (1.5) of Tob1 to distinguish colon cancer from normal tissues $(P<0.001$, Fig. 1F). The point on the curve was close to $(0.0,1.0)$, maximizing both sensitivity $(97.6 \%)$ and specificity (71.4\%).

Additional file 1: Table S2 shows that high Tob1 expression was significantly associated with tumour size $(P=0.035)$ and tumour differentiation $(P=0.000)$. Kaplan-Meier analysis of the mean OS showed reduced survival in patients with high Tob1 expression (55.0 months) compared to those with low Tob1 expression (82.1 months) $(P=0.029$, Fig. 1G). Univariate analysis indicated that Tob1 expression, TNM stage, lymphatic metastasis and tumour size were significant prognostic factors for OS (Additional file 1: Table S3). Nevertheless, Tob1 expression was not an independent prognostic factor for OS in multivariate analysis. A longer OS was also observed in stage I-II colon cancer patients with low Tob1 expression than in those with high Tob1 expression $(P=0.023$, Fig. $1 \mathrm{H})$. Univariate and multivariate analyses showed that Tob1 expression $(P=0.012)$ and lymphovascular invasion $(P=0.003)$ were independent prognostic indicators for OS in stage I-II colon cancer patients (Additional file 1: Table S4). 


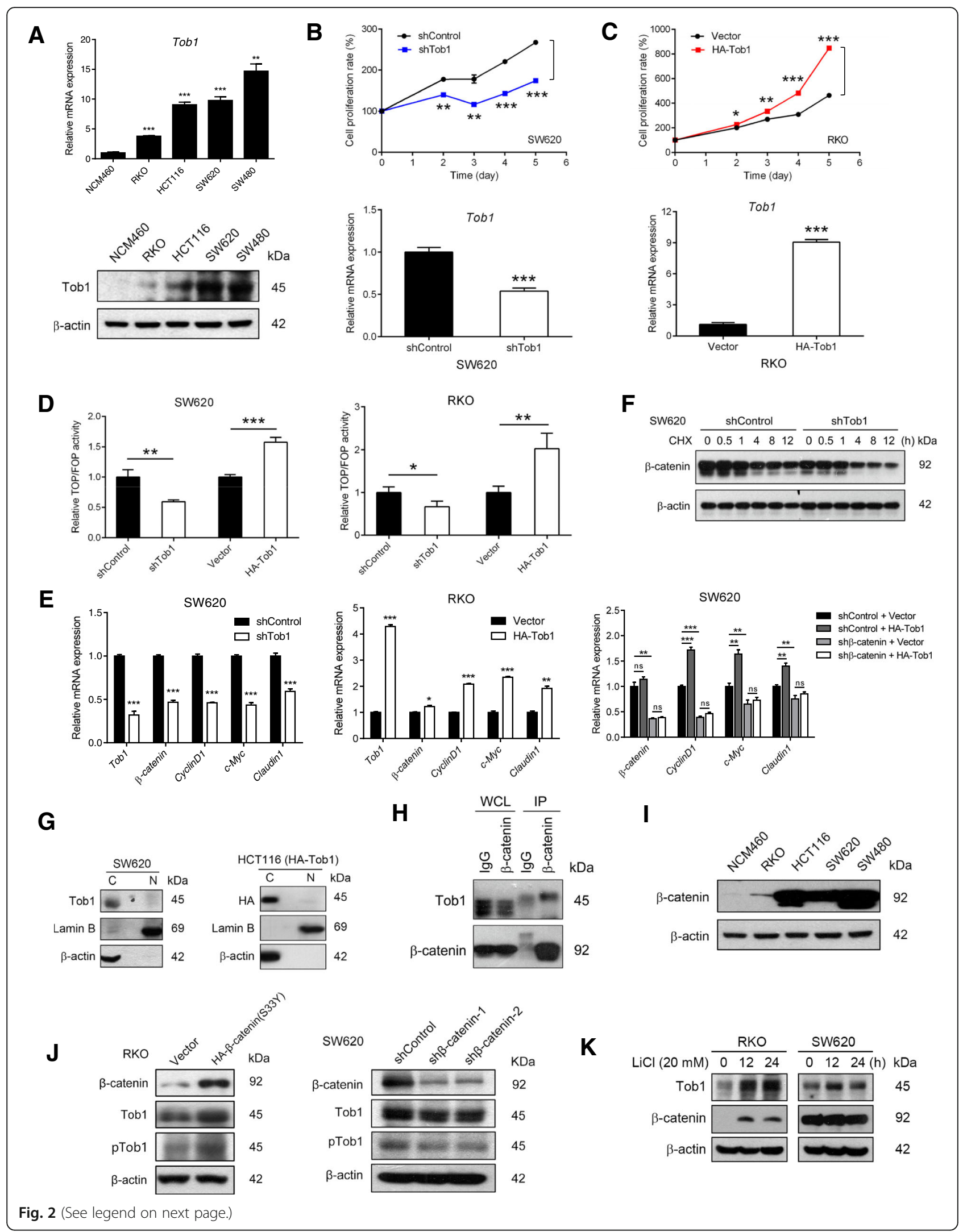


(See figure on previous page.)

Fig. 2 Tob1 promotes colon cancer cell proliferation via a Wnt/B-catenin signalling feedback loop. a Tob1 expression analysis by qRT-PCR and WB. b-c Effects of Tob1 knockdown or overexpression on cell proliferation analysed by MTT. $\mathbf{d}$ Wnt activity analysis by the TOPFlash/FOPFlash assay. e mRNA expression analysis of Wnt/ $\beta$-catenin target genes by qRT-PCR. $\mathbf{f} \beta$-Catenin levels in cells treated with $\mathrm{CHX}(10 \mu \mathrm{g} / \mathrm{ml})$. $\mathbf{g}$ Subcellular localization analysis of Tob1 by nucleocytoplasmic separation. $\mathbf{h}$ Interaction analysis of $\beta$-catenin and Tob1 by co-IP. i $\beta$-Catenin expression analysis by WB. $\mathbf{j} \beta$-Catenin regulated the expression but not phosphorylation of Tob1. $\mathbf{k}$ Tob1 expression was upregulated by LiCl in RKO cells. ${ }^{*}, P<0.05 ;{ }^{*}, P<0.01$ and ${ }^{* *}, P<0.001$ vs control

Tob1 promotes colon cancer cell proliferation via a Wnt/ $\beta$-catenin signalling positive feedback loop in vitro

Because Tob1 expression was associated with tumour size but not tumour invasion depth or lymph node metastasis, we analysed the involvement of Tob1 in colon cancer cell growth. The mRNA and protein levels, but not phosphorylation (normalized to expression), of Tob1 were much higher in colon cancer cells than in normal colonic epithelial NCM460 cells (Fig. 2a \&
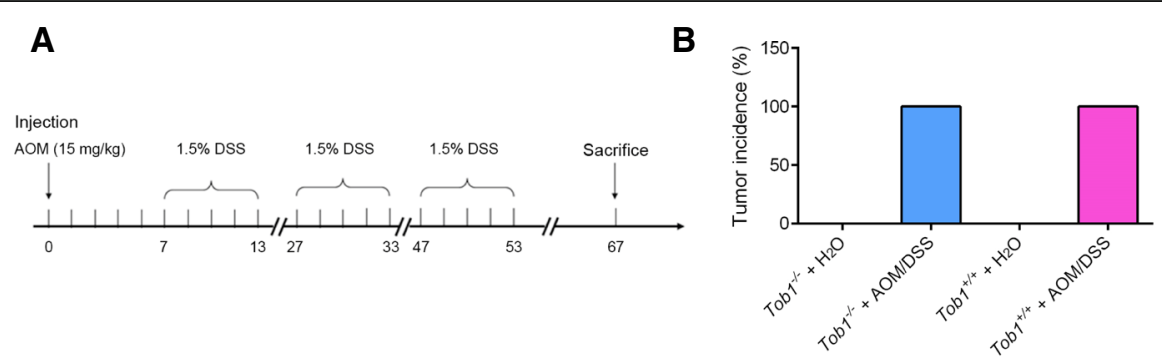

C

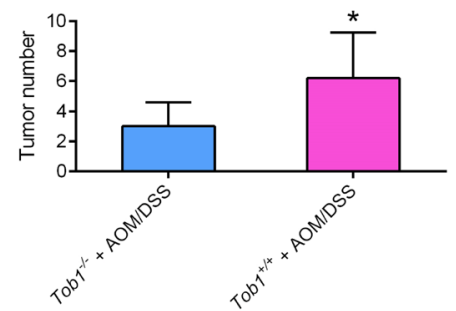

$\mathbf{E}$

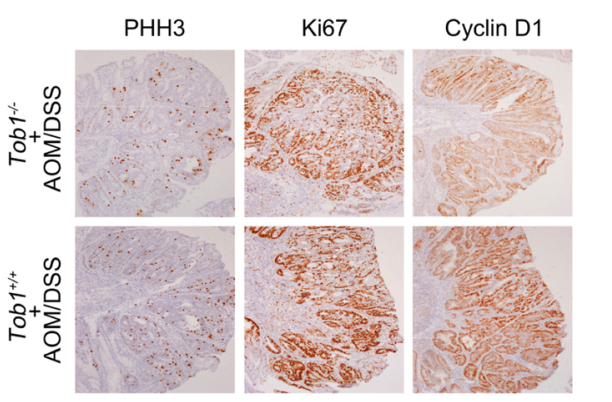

G

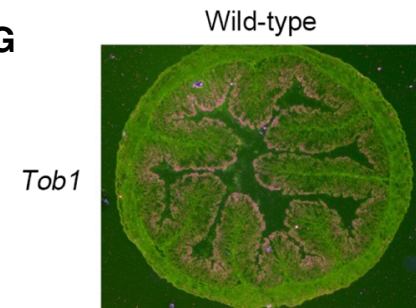

D

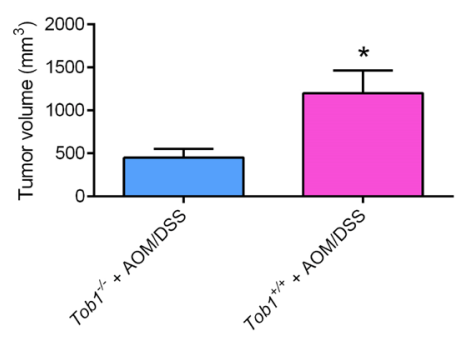

$\mathbf{F}$

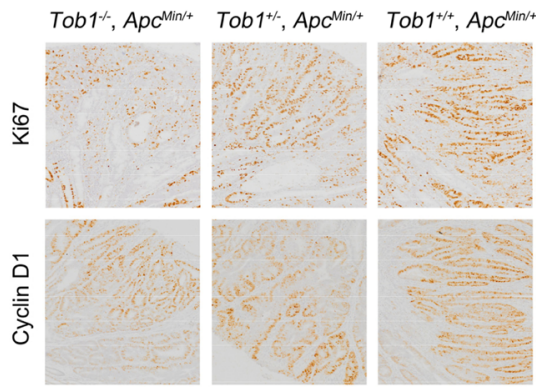

$\mathrm{Apc}^{\mathrm{Min} /+}$

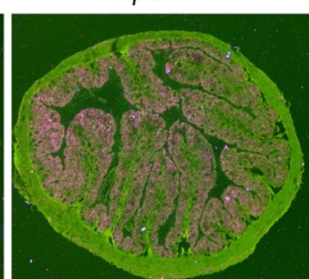

Fig. 3 Tob1 stimulates proliferation and Wnt signalling in vivo. a Experimental procedures for the AOM/DSS model. b-d Colon tumour incidence, number and volume in mice. ${ }^{*}, P<0.05$. e-f Representative $\mathrm{HHC}$ micrographs of $\mathrm{PHH}, \mathrm{Ki}-67$ and cyclin D1 in colon tumours of different mouse models. $\mathbf{g}$ ISH analysis of Tob1 expression in the colon tissues of $\mathrm{ApC}^{\mathrm{Min} /+}$ mice 
Additional file 1: Figure S3). Tob1 knockdown dramatically decreased the growth of high-Tob1-expressing SW620 cells, while overexpression of Tob1 significantly promoted the proliferation of low-Tob1-expressing RKO cells (Fig. 2b-c), indicating a role for Tob1 in the promotion of colon cancer cell growth.

Due to the pivotal role of dysregulated $\mathrm{Wnt} / \beta$-catenin signalling in colon cancer development [8], we asked whether this pathway contributes to the effect of Tob1 on colon cancer. Tob1 knockdown significantly inhibited and Tob1 overexpression greatly enhanced $\beta$-catenindependent transcriptional activity in SW620 and RKO cells, respectively (Fig. 2d). Moreover, the mRNA levels of Cyclin D1, c-Myc and Claudin1, important Wnt/ß-catenin target genes to regulate cell proliferation, changed significantly with Tob1 expression in SW620 and RKO cells in a $\beta$-catenin-dependent manner (Fig. 2e). We then investigated the modulation of $\beta$-catenin signalling by Tob1. Given that the turnover of cytoplasmic $\beta$-catenin is a central event [8], we tested whether Tob1 could influence $\beta$-catenin stability. As expected, Tob1 knockdown led to rapid degradation of $\beta$-catenin under $\mathrm{CHX}$ treatment in SW620 cells (Fig. 2f). To understand how Tob1 stabilized $\beta$-catenin, the unique cytoplasmic location observed in Fig. 1D was confirmed in SW620 and HCT116 cells (Fig. $2 \mathrm{~g})$. We found that Tob1 interacted with $\beta$-catenin in RKO cells expressing high levels of $\beta$-catenin due to pre-treatment with the GSK3 inhibitor CHIR99021 $(6 \mu \mathrm{M}, 24 \mathrm{~h})$ (Fig. 2h). These results suggested that Tob1 interacts with $\beta$-catenin in the cytoplasm and increases $\beta$-catenin stability, thereby upregulating $\beta$-catenin signalling and promoting colon cancer cell growth.

We then explored whether hyperactive Wnt signalling in colon cancer [8] inversely contributed to upregulated Tob1 expression. $\beta$-Catenin expression was correlated with Tob1 expression in four colon cancer cell lines (Fig. $2 a$, Fig. 2i). Moreover, $\beta$-catenin overexpression elevated and $\beta$-catenin knockdown reduced Tob1 expression in RKO and SW620 cells, respectively (Fig. 2j). Tob1 expression was also upregulated by $\mathrm{LiCl}$-induced activation of Wnt/ß-catenin signalling in RKO cells but not SW620 cells, which are unable to respond to $\mathrm{LiCl}$ owing to APC mutation [9] (Fig. 2k). Tob1 phosphorylation, when normalized to expression, was not noticeably changed by modulation of $\beta$-catenin levels in RKO and SW620 cells (Fig. 2j \& Additional file 1: Figure S4). The above patterns suggest a positive feedback loop between Tob1 expression and Wnt signalling in the progression of colon cancer.

\section{Tob1 deficiency leads to reduced tumourigenesis and malignant tendencies in AOM/DSS-treated mice and $\mathrm{Apc}^{\mathrm{Min} /+}$ mice}

Tob1 knockdown was further performed in two mouse models of colon cancer-a drug-induced model (AOM/
DSS treatment) and gene-disrupted model ( $A p c^{\mathrm{Min} /}$ $\left.{ }^{+}\right)$- to explore the role of Tob1 in vivo. After 3 rounds of DSS exposure following AOM injection (Fig. 3a), $\mathrm{Tob1}^{-/-}$and $\mathrm{Tobl}^{+/+}$mice exhibited $100 \%$ incidence of

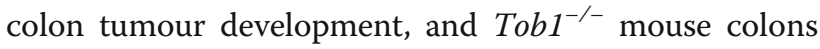
exhibited fewer AOM/DSS-induced tumours than $\mathrm{Tobl}^{+/+}$mouse colons (Fig. 3b-d). Tob1 deficiency resulted in reduced cell proliferation, based on the levels of Ki67 and PHH3, two markers of cell proliferation. In addition, the downstream target protein of the Wnt signalling pathway, cyclin D1, was also repressed in the absence of Tob1 (Fig. 3e). Consistent with this finding, colorectal tumours in $\mathrm{Tob1}^{-/-}, \mathrm{Apc}^{\mathrm{Min} /+}$ mice had the lowest malignant tendencies among these three groups, while $\mathrm{Tobl}^{+/+}, \mathrm{Apc}^{\mathrm{Min} /+}$ mice developed the most malignant tumours (Fig. 3f). In situ hybridization analysis further indicated upregulation of Tob1 expression in $A p c^{\mathrm{Min} /+}$ mice with overactivated Wnt signalling pathways (Fig. $3 g$ ). These in vivo results were consistent with the in vitro results, indicating that Tob1 and Wnt signalling are mutually regulated to promote colon cancer development.

\section{Conclusion}

Here, we identified a unique oncogenic role of Tob1 as an adverse prognostic factor for colon cancer, where Tob1 is localized mainly in the cytosol and promotes cell growth via a Wnt positive feedback loop.

\section{Additional file}

Additional file 1: Supplementary Information. (DOCX $260 \mathrm{~kb}$ )

\section{Abbreviations}

AOM: Azoxymethane; APC: Adenomatous polyosis coli; AUC: Area under the curve; CHX: Cycloheximide; co-IP: Co-immunoprecipitation; DSS: Dextran sodium sulphate; GSK3: Glycogen synthase kinase 3; HR: Hazard ratio; IHC: Immunohistochemistry; IP: Immunoprecipitation; ISH: In situ hybridization; Min: Multiple intestinal neoplasia; mTOR: mammalian target of rapmycin; OS: Overall survival; $\mathrm{PHH} 3$ : Phosphohistone H3; qRT-

PCR: Quantitative reverse-transcription PCR; ROC curve: Receiver operating characteristic curve; SD: Standard deviation; TCGA: The Cancer Genome Atlas; TMA: Tissue microarray; TOB1: The transducer of ERBB2.1; WB: Western blotting; WCL: Whole cell lysate

\section{Acknowledgements}

This work was supported by the National Natural Science Foundation of China, No. 81472568 (to T.H.), U1405228 (to T.H.), 31770860 (To T.H.) and 81572589 (to Y.-Y.Z.); the Natural Science Foundation of Fujian Province, No. 2017 J06020 (to Y.-Y.Z.), 2017R1036-4 (to Y.-Y.Z.), 2016R1034-4 (to W.Z.), 2016R1034-1 (to H.C.), 2017R1036-6 (to B.C.) and 2018 J01400 (to L.X.), and the Health and young middle-aged of personnel training project funding of Fujian Province No. 2016-ZQN-89 (to L.X.).

Funding

Not applicable.

Availability of data and materials Additional file 1 was available online. 


\section{Author's contributions}

Concept and design: HW, YZ, TH; Performing experiments: DL, LX, YG, YF, BC Analysis of data: $\mathrm{DL}, \mathrm{LX}$; Contributing reagents, materials and other analytical tools: YG, WZ, HC; Writing the manuscript: TH, YZ, LX. All authors read and approved the final manuscript.

\section{Ethics approval and consent to participate}

A total of 109 specimens from colon cancer patients were used for this study, which was approved by the Medical Ethics Committee of Zhongshan Hospital Affiliated to Xiamen University in accordance with the Helsinki Declaration and conducted with the informed consent of all patients. All animals were performed in accordance with a protocol approved by the Animal Care and Use Committee of Xiamen University.

\section{Consent for publication}

This article is distributed under the terms of the Creative Commons Attribution 4.0 International License (http://creativecommons.org/licenses/by/ 4.0/), which permits unrestricted use, distribution, and reproduction in any medium, provided you give appropriate credit to the original author(s) and the source, provide a link to the Creative Commons license, and indicate if changes were made. The Creative Commons Public Domain Dedication waiver (http://creativecommons.org/publicdomain/zero/1.0/) applies to the data made available in this article, unless otherwise stated.

\section{Competing interests}

The authors declare that they have no competing interests.

\section{Publisher's Note}

Springer Nature remains neutral with regard to jurisdictional claims in published maps and institutional affiliations.

\section{Author details}

'Xiamen Cancer Hospital, The First Affiliated Hospital of Xiamen University, Xiamen 361003, Fujian Province, People's Republic of China. ${ }^{2}$ Cancer Research Center, Xiamen University Medical College, Xiamen 361102, Fujian Province, People's Republic of China. ${ }^{3}$ Department of Oncology, Zhongshan Hospital Affiliated to Xiamen University, Xiamen 361004, Fujian Province, People's Republic of China. ${ }^{4}$ Health and family planning commission of Huai'an city, Huai'an 223000, Jiangsu Province, People's Republic of China. ${ }^{5}$ Reproductive Medical Center, The First Affiliated Hospital of Xiamen University, Xiamen 361003, Fujian Province, People's Republic of China. ${ }^{6}$ Fujian Provincial Key Laboratory of Reproductive Health Research, Xiamen University Medical College, Xiamen 361102, Fujian Province, People's Republic of China.

Received: 20 April 2018 Accepted: 1 November 2018 Published online: 17 November 2018

\section{References}

1. Kundu J, Wahab SM, Kundu JK, Choi YL, Erkin OC, Lee HS, Park SG, Shin YK. Tob1 induces apoptosis and inhibits proliferation, migration and invasion of gastric cancer cells by activating Smad4 and inhibiting betacatenin signaling. Int J Oncol. 2012;41:839-48.

2. Kawamura-Tsuzuku J, Suzuki T, Yoshida Y, Yamamoto T. Nuclear localization of Tob is important for regulation of its antiproliferative activity. Oncogene. 2004;23:6630-8.

3. O'Malley S, Su H, Zhang T, Ng C, Ge H, Tang CK. TOB suppresses breast cancer tumorigenesis. Int J Cancer. 2009;125:1805-13.

4. Zhang YW, Nasto RE, Varghese R, Jablonski SA, Serebriiskii IG, Surana R, Calvert VS, Bebu I, Murray J, Jin L, et al. Acquisition of estrogen independence induces TOB1-related mechanisms supporting breast cancer cell proliferation. Oncogene. 2016;35:1643-56.

5. Fussbroich B, Wagener N, Macher-Goeppinger S, Benner A, Falth M, Sultmann H, Holzer A, Hoppe-Seyler K, Hoppe-Seyler F. EZH2 depletion blocks the proliferation of colon cancer cells. PLoS One. 2011;6:e21651.

6. Rhodes DR, Yu J, Shanker K, Deshpande N, Varambally R, Ghosh D, Barrette T, Pandey A, Chinnaiyan AM. Large-scale meta-analysis of cancer microarray data identifies common transcriptional profiles of neoplastic transformation and progression. Proc Natl Acad Sci U S A. 2004;101:9309-14.

7. Helms MW, Kemming D, Contag CH, Pospisil H, Bartkowiak K, Wang A, Chang SY, Buerger H, Brandt BH. TOB1 is regulated by EGF-dependent HER2 and EGFR signaling, is highly phosphorylated, and indicates poor prognosis in node-negative breast cancer. Cancer Res. 2009;69:5049-56.

8. Gregorieff A, Clevers H. Wnt signaling in the intestinal epithelium: from endoderm to cancer. Genes Dev. 2005;19:877-90.

9. Almeida A, Bolanos JP, Moncada S. E3 ubiquitin ligase APC/C-Cdh1 accounts for the Warburg effect by linking glycolysis to cell proliferation. Proc Natl Acad Sci U S A. 2010;107:738-41.

\section{Ready to submit your research? Choose BMC and benefit from:}

- fast, convenient online submission

- thorough peer review by experienced researchers in your field

- rapid publication on acceptance

- support for research data, including large and complex data types

- gold Open Access which fosters wider collaboration and increased citations

- maximum visibility for your research: over $100 \mathrm{M}$ website views per year

At BMC, research is always in progress.

Learn more biomedcentral.com/submissions 